\title{
CLOUD-BASED APPLICATION FOR SMART GRID SIMULATION
}

\author{
SZABOLCS TAR ${ }^{1 *}$ AND ATTILA FODOR ${ }^{2}$ \\ ${ }^{1}$ Delta-Group Holding Zrt., Szentendrei út 39-53, Budapest, 1033, HUNGARY \\ 2 Department of Electrical Engineering and Information Systems, University of Pannonia, \\ Egyetem út 10, Veszprém, 8200, HUNGARY
}

\begin{abstract}
This study provides an overview of the content of an R\&D project for increasing the efficiency of smart grids to analyse the energy market, consumption, generation, and renewable energy usage. The new energy participants, for example decentralized generation, dynamic consumption, and weather-dependent power plants, are facing challenges. This project offers solutions to these challenges: modelling of distribution networks, forecasts of renewable energy sources distributed generation (RES-DG), micro-grid management and responses to demand in new, complex solutions. A hardware device is being developed for smart grids and security modules to enhance IT security.
\end{abstract}

Keywords: renewable energy, smart grid, demand response, cyber-security, network assets

\section{Introduction}

The main objective of our project is to utilize the opportunities in smart grids based on smart grid data and remote control to improve energy and cost efficiency. The service package is composed in a way that our solutions are put into use either as a whole or for the fulfilment of subtasks. The services and hardware devices are being developed like one intelligent cloud-based service.

The rationale of these solutions is supported by several aspects of the energy market. The vastly proliferating renewable energy sources distributed generation producers (RES-DG) are affecting every player in the energy market. The number and built-in power of such power stations are expected to exhibit a rising tendency in the near future according to various energy tenders, see Tables 1 and 2.

\section{Experimental Data}

Grid operators should find a solution for operating grids with more entry points replacing the ones utilizing one directional power flow, where the direction of power flow changes over time and is influenced mainly by the performance of weather-dependent power plants.

Fig.1 shows the electricity consumption of Hungary, which displays both domestic generation and importation, as well as planned and actual values of power transmissions from or to neighbouring countries. The prime cost of the electricity and the volume of the consumption set the actual, base, and peak prices of

*Correspondence: Szabolcs.Tar@Delta.hu
Table 1. Number of (pc.) and power generation (kW/MW) of RES-DG producers over recent years in Hungary [1].

\begin{tabular}{lcccccc}
\hline & \multicolumn{2}{c}{2008} & \multicolumn{2}{c}{2014} & \multicolumn{2}{c}{$2015^{*}$} \\
& pc. & $\mathrm{kW}$ & pc. & MW & pc. & MW \\
\hline Solar & 107 & 363 & $8.8 \mathrm{k}$ & 68 & $11 \mathrm{k}$ & 84 \\
Wind & 1 & 10 & 56 & 0.5 & 56 & 0.5 \\
Hydro & 1 & 16 & 3 & 0.06 & 3 & 0.06 \\
\hline
\end{tabular}

Table 2. Use of renewable energy in Hungary [2].

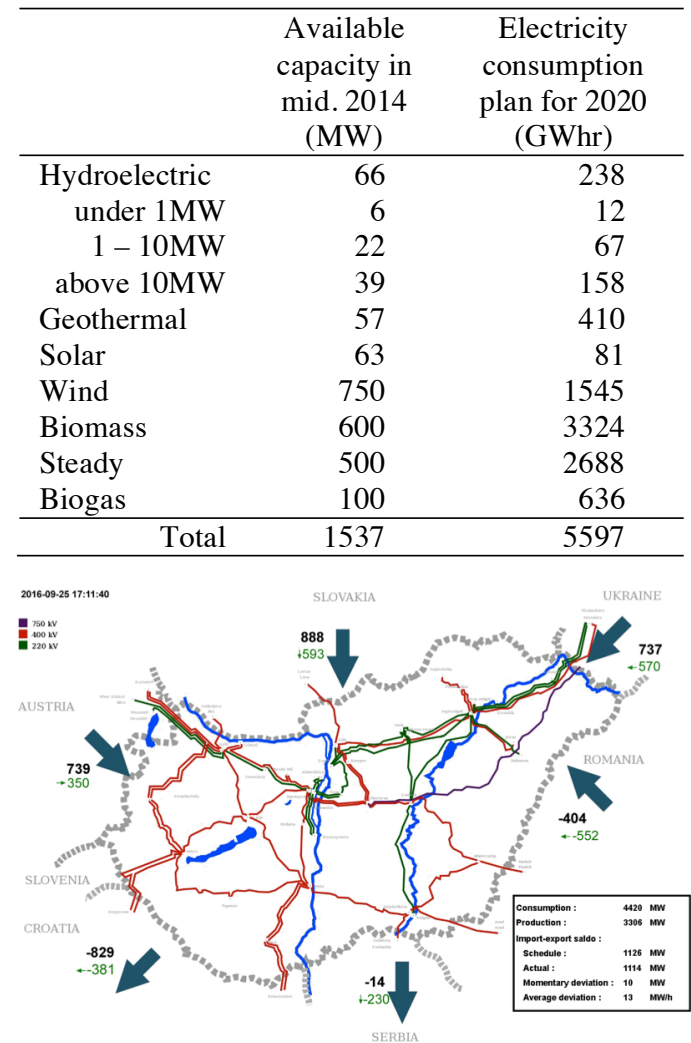

Figure 1. Electricity consumption, export, and import of Hungary as of September 25, 2016 (at 5 p.m.) [1]. 


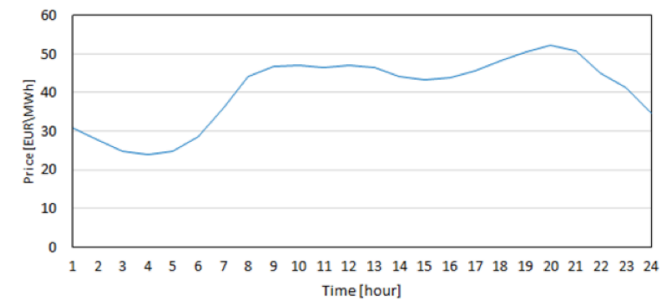

Figure 2. Hourly average price of electrical energy in Hungary (2015).

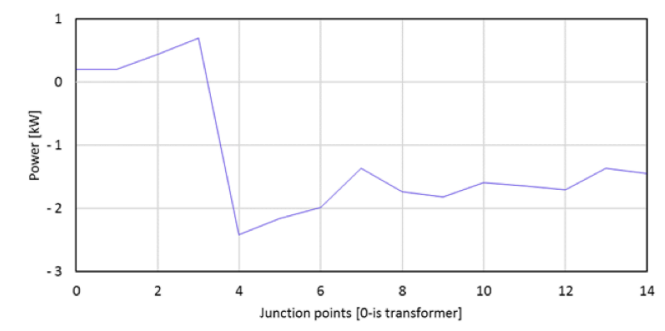

Figure 4. Consumption of the electricity network of consumers (power versus 15 minute long sample times).

electrical energy. Fig.2 shows the hourly average price of the electrical energy in 2015 in Hungary. As solar and wind renewable energy sources are somewhat uncontrollable, the integration of renewable sources is a big challenge for transmission operators.

As a solution, our group implemented a calculation model of the grid that takes into account parameters of the grid elements and details of network measurements. Details about grids are provided by smart measurement and points of consumption defined in the remote reading circuit. Measurement data are generated over periods of at least 15 minute in length at these measurement points.

\section{Simulations}

The spread of decentralised energy producers based on Table 1, cause time-dependent power flows in grid sections. Figs.3-5 show the load of a radial grid examined at various times. The lines on the left side of the graphs start from the contact bar of a transformer, the specific points show the power of grid lead-downs. Positive values indicate a flow of power from a transformer towards an endpoint of the grid, while negative values illustrate a flow of power from an endpoint of the grid towards a transformer.

\section{Results and Discussion}

It is reasonable according to the graphs that the built-in power is insufficient but the trends of consumption and production should be taken into account and a mathematical model created to know the exact operating states as well as for the integration requirement of the new production unit.

In order to be able to model the integration of new RES-DG efficiently and safely over a specific section,

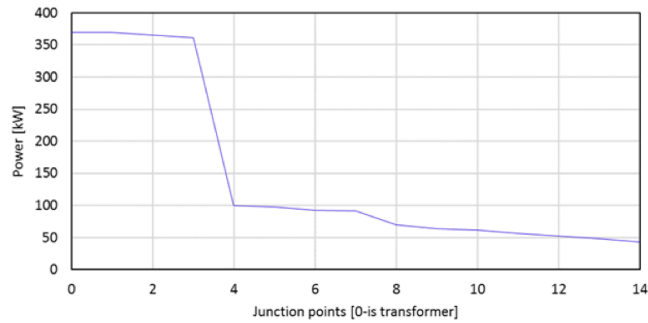

Figure 3. Consumption on the electricity network of consumers only (power versus number of 15 minute sample times).

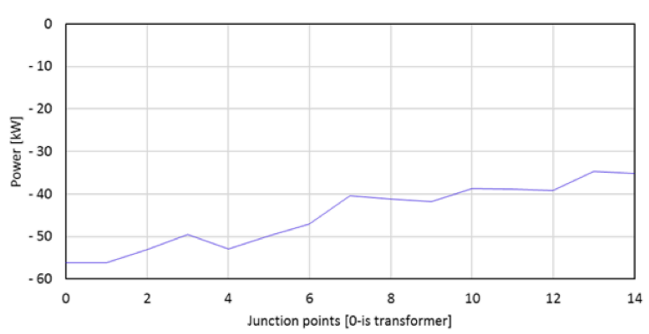

Figure 5. Consumption on the electricity networof consumers (power versus 15 minute long sample times).

new forecast services need to be impremented. Our service concept is mainly based on mass useage of solar cells in accordance with Table 1. External environmental aspects were taken into account that affect the operation of renewable energy source power stations such as clouds, the temperature, humidity, global isolation as well as characteristics of power stations, such as orientation, tilt angle, integrated power, type and efficiency. The sources of data are defined, e.g. online satellite images and analytics, which in a complex fashion, can be used to create forecasts based on factual measurement data.

For microgrid operators, e.g. low- and mediumvoltage operators, private grid operators, industrial parks, multiple household buildings, balancing groups, etc., a complex energy service concept was outlined. in which an opportunity for intervention was provided to facilitate a more efficient operation examining the production and consumption habits involved in the given microgrid. If the green energy produced is to be used as widely as possible, it should be identified where and to what extent the available green energy was consumed. By utilizing storage capacities, energy market transactions should be performed that are regarded as new nowadays, e.g. electrical energy is sold and thermal and hydrogen energy sources, vehicle charging, or other services purchased. Our group has proposed a support application service for this. Furthermore, settlement and cost allocation becomes more efficient and clearer based on details for operators. Meters and sensors as well as intervention devices allow microgrid operators to modify the image of a grid proactively based on up-to-date information reducing service outage, which is highly relevant not only for consumers but also producers and trade licence holders too, as an outage location impacting both consumption plan and production plans. An outage location leads to 
unplanned resource withdrawal due to the spread of RES-DG power stations. Microgrid providers can initiate interventions to influence the expected production and consumption, providing basic parameters of energy usage. This basic signal can be a plan or request to balance out deviation in the plan. The controller is a decision logics based on the deviation of measurements and basic signals that issues a command within the available limits, which is based on a complex logics by transferring this to the energy use and consumption process in order to achieve the desired result.

The consumer and equipment of the consumer are parts of the energy-use and -production processes (hereinafter referred as to process), respectively. This equipment is connected to smart grids among others via smart home devices. To allow the consumer to interact, a service shall be developed that is users demand. When intervention in the process does not take place automatically, the consumer is provided with detailed and aggregated information via various solutions in which the result of his/her intervention is displayed as well as the decrease or rise in energy use or costs.

The means of automatic intervention or interaction are dynamic tariffs, which also require smart grids that provide detailed and instantly available data. This allows for the service provider supplying dynamic tariffs to make an offer that reaches the user in a timely manner, which will be either reviewed or accepted by the user or just received as information, as it is possible to intervene remotely and automatically in processes. The goal of our service is to make this information available on a platform based on priorities, irrespective of service providers, ensuring the opportunity to change of energy provider and the access previous data. We provide information to consumers as well as producers by connecting the initiator of an intervention securely via processes about which information, states, and backtesting are also generated by us to providers.

To ensure the overall security of smart grids and services included in smart grids, the situation of cyber security and its expectations shall be mapped. Smart grids are regarded as an extended electronic information system, whose endpoints can be accessed and managed remotely. However, they are less verified and have partial physically protection. The main requirements considered are

- Act. CXII of 2011 on the right of information selfdetermination and information liberty;

- Act. CLXVI of 2012 on the identification, designation and protection of essential systems and facilities;

- Act. L of 2013 on the electronic information security of state- and local government-owned organisations;

- Directive 95/46/EC of the European Parliament and of the Council

- Relevant provisions of energy legislation

- Information methodologies and recommendations
A hardware development has been implemented to enhance information security and a universal communication device has likewise been developed in the framework of hardware development offering a solution that facilitates efficient communication between various devices of smart grids.

\section{Conclusion}

These services and hardware devices reduce the costs for the participants in the energy sector from generation to consumption via distribution networks, and provide more secure ITC infrastructure. To manage and forecast generation and consumption, new implementations of RES-DG can be easily installed in the current distribution network, without the need for extra investment. The service package is flexible. Anyone can choose whether to use services in the future or logged date. The system will be extended to include other new services.

Such services generate data requirements. Further complex analytics provide useful results for the value chain actors, e.g. to improve the reliability of the distribution network. A source of irregular consumption is unchecked loads on the network, which leads to the overload of built-in elements of infrastructure, premature aging and damage. The simulation engine analyses the data and generate alerts concerning the status of network elements. Before a failure occurs, the system can take various actions to reduce its duration and damage caused in order to expand cloud-based services for industrial consumers to dynamically control consumption for the network balancing. As far as the value chain is concerned, actors provide further bonuses to reduce energy costs.

The devices and services in smart grids, like in telecommunications or IT infrastructure create an opportunity for the further integration of sensors and surveillance camera networks. This integration into the management systems of cities is currently being tested.

\section{Acknowledgement}

We acknowledge the financial support of this work by the Hungarian State under the VKSZ_12-1-2013-0088 project.

\section{REFERENCES}

[1] Hungarian energy and public utility regulatory authority (MEKH), 2015 www.mekh.hu/nemengedelykoteles-kiseromuvek-es-haztartasi-meretukiseromuvek-adatai-2008-2015-villamos-energia

[2] Hungarian Independent Transmission Operator Company Ltd. (MAVIR) analysis of the Hungarian electricity network, 2015 www.mavir.hu/documents/ 10258/15461/Forr\%C3\%A1selemz\%C3\%A9s_2015.pdf

[3] Hungarian Power Exchange Ltd. (HUPX): www.hupx.hu 